\title{
Modeling and numerical simulation of thermal ageing in a filled rubber
}

\author{
K.D. Ahose \& S. Lejeunes \& D. Eyheramendy \\ Aix-Marseille Univ, CNRS, Centrale Marseille, LMA UMR7031, F-13402 Marseille Cedex 20, France \\ R. Bouaziz \\ Mines ParisTech, Centre des Matériaux, CNRS UMR7633, F-91003 Evry Cedex, France
}

\begin{abstract}
In this work, we propose a constitutive model for the representation of thermal ageing phenomena in a butadiene rubber filled with carbon blacks. This model is based on a thermo-chemo-mechanical framework and takes into account the strong couplings that occur during ageing. This model has been implemented in a multi-fields finite element framework. Based on simple experimental tests, we discuss the identification of material parameters and more particularly the chemo-mechanical coupling term which is identified from inverse finite element simulations.
\end{abstract}

\section{INTRODUCTION}

In a previous work (see Ahose, Lejeunes, Eyheramendy, \& Sosson 2017), we presented some experimental results obtained from thermal ageing tests (mainly under anaerobic conditions) carried out on a butadiene rubber filled with carbon blacks (see also Ahose 2018). Tensile samples were placed in a oven at differents temperatures $\left(50^{\circ} \mathrm{C}, 70^{\circ}, 90^{\circ}\right)$ for different ageing times (1 to 29 days) and we sytematically performed mechanical and swelling tests to investigate the consequence of thermal ageing on these samples. The main mechanism that was observed was a maturation (i.e. an increase of the apparent crosslink density) of the crosslinked network. This effect results in an increase in the material's stiffness, a decrease in its ability to dissipate mechanical energy and a decrease in the strength limit. By proceeding in a same way with cylindrical samples, we also observed that thermal ageing does not affect hydrostatic behavior (at least for the time and temperature of ageing that were considered in our study). By performing ageing tests under permanent mechanical load (creep ageing test), we studied the coupling between the mechanical state and the ageing process. We found that, for the studied material, a permanent tensile load plays a role on the evolution of the crosslinked network (maturation was lower in areas where the mechanical loading was higher). This result highlights a chemo-mechanical coupling effect but surprisingly, this effect does not seem to induce anisotropy (we performed mechanical tests in different direction after ageing) and do not involve a specific permenant set due to the chemical process when the mechanical load is removed (more precisely permenant set was found to be nearly identical with or without thermal ageing).

All these observations guided us in the developpement of a fully coupled thermo-chemo-mechanical constitutive model. The general constitutive framework was developped in Lejeunes, Eyheramendy, Boukamel, Delattre, Méo, \& Ahose 2018, Lejeunes \& Eyheramendy 2018. We describe ageing by introducing a dimensionless, scalar, internal variable that is related to the observed variation in apparent crosslink density. This phenomenological modeling of ageing is also proposed by other authors (e.g. Kari 2017, Musil, Johlitz, \& Lion 2018). For the mechanical part, we reused the works of Delattre, Lejeunes, Méo, Lacroix, \& Richard 2014, Delattre, Lejeunes, Lacroix, \& Méo 2016 done on the same material in the virgin state. The mechanical model takes into account a thermomechanical coupling and of the Payne effect.

The originality of the current model lies in the chemical evolution law and its coupling with mechanics. We propose to briefly describe the model and focus on the material parameter identification for which we used homogeneous and non-homgeneous tests. For the last category mentionned, we used finite element simulations as part of an inverse indentification strategy.

\section{CONSTITUTIVE MODELING}

First, we define $\xi(\mathbf{x}, t)$ as the local chemical conversion (dimensionless parameter) such as $\xi \in[0,1]$ and $\xi(\mathbf{x}, 0)=0$. This internal variable accounts for chemical modification of the crosslinked network during 
ageing and we related it directly to the increase in the apparent crosslink density with the relation:

$\xi(\mathbf{x}, t)=\frac{Q(\mathbf{x}, t)-Q_{0}(\mathbf{x}, 0)}{Q_{\infty}(\mathbf{x}, \infty)-Q_{0}(\mathbf{x}, 0)}$

where $Q$ is the inverse of the swelling ratio ${ }^{1}, Q_{0}$ is the inverse of the initial swelling ratio and $Q_{\infty}$ is the maximum value of $Q$ that the material can reach.

By assuming the following decomposition of the deformation gradient $\mathbf{F}$ :

$\mathbf{F}=J^{-1 / 3} \overline{\mathbf{F}}_{\mathbf{e}}^{i} \overline{\mathbf{F}}_{\mathbf{v}}^{i}, \quad i=1 . . N$

we introduce $N$ internal variables to account of viscous effect. As we do not observe a coupling between thermal aging and the volumetric behavior, we assume that the volume variation can be only due to two contributions: thermal dilatation and mechanical compressibility, therefore we have:

$J=J_{\Theta} J_{m}, J_{\Theta}=1+\alpha_{\Theta}\left(\Theta-\Theta_{0}\right), J_{m}=J / J_{\Theta}$

To account of a dynamical softening effect (Payne effect) we also consider $n$ supplementary dimensionless internal variables, $\omega_{i} \in[0,1]$ (see for instance Delattre, Lejeunes, Lacroix, \& Méo 2016).

\subsection{Thermodynamics and constitutive relations}

We adopt the formalism with the hybrid free energy (see Lejeunes, Eyheramendy, Boukamel, Delattre, Méo, \& Ahose 2018). The hybrid free energy is assumed as follows:

$$
\begin{aligned}
\varphi\left(\overline{\mathbf{B}}, \overline{\mathbf{B}}_{\mathbf{e}}^{i}, \omega_{i}, q, \xi, \Theta\right) & =\varphi_{\text {iso }}\left(\overline{\mathbf{B}}, \overline{\mathbf{B}}_{\mathbf{e}}^{i}, \omega_{i}, \xi, \Theta\right) \\
& +\varphi_{\text {vol }}(q, \Theta)+\varphi_{\text {therm }}(\Theta) \\
& +\varphi_{\text {chem }}(\xi, \Theta)
\end{aligned}
$$

where $\overline{\mathbf{B}}=\overline{\mathbf{F}} \cdot \overline{\mathbf{F}}^{\mathrm{T}}$ and $\overline{\mathbf{B}}_{\mathbf{e}}^{i}=\overline{\mathbf{F}}_{\mathbf{e}}^{i} \cdot \overline{\mathbf{F}}_{\mathrm{e}}^{i^{\mathrm{T}}}$ are the isochoric left Cauchy Green tensor and the $i^{\text {th }}$ elastic isochoric left Cauchy Green tensor. The variable $q$ is a pressure like variable. The additive splitting of eq. (4) is motivated by the fact that there can exist purely thermal variations that are stress-free for which no chemical reaction occurs (contribution of $\left.\varphi_{\text {therm }}\right)$. Themo-chemical evolutions that are stressfree can also be observed (contribution $\varphi_{\text {chem }}$ ) and finally thermo-mechanical states can also exist without any chemical reactions occurring. Furthermore, due to the huge difference in stiffness for hydrostatic or isochoric states we adopt an isochoric/volumetric split for the remaining part of the hybrid energy (contributions of $\varphi_{i s o}$ and $\varphi_{v o l}$ ). From the experiments,

\footnotetext{
${ }^{1}$ The inverse of the swelling ratio (capacity of an elastomer to swell in a solvent) can be related to the apparent crosslink density with the Flory-Rehner theory of swelling.
}

we do not observe impact of the aging on the thermal properties of the material (heat capacity and thermal dilatation), therefore $\varphi_{\text {therm }}$ is assumed to depend only on $\Theta$. It is the same for the volumetric contribution, therefore $\varphi_{v o l}$ depends only on the pressure-like variable $q$ and the temperature.

Using the previously defined hybrid energy, the Clausius-Duhem inequality takes the following form:

$$
\begin{gathered}
\phi=\left(\boldsymbol{\sigma}-2 \rho\left(\overline{\mathbf{B}} \frac{\partial \varphi}{\partial \overline{\mathbf{B}}}+\sum_{i=1}^{N} \overline{\mathbf{B}}_{\mathbf{e}}^{i} \frac{\partial \varphi}{\partial \overline{\mathbf{B}}_{\mathbf{e}}^{i}}\right)^{\mathrm{D}}-\frac{q}{J_{\Theta}} \mathbf{1}\right): \mathbf{D} \\
-\rho\left(s+\frac{\partial \varphi}{\partial \Theta}-\frac{q J \alpha_{\Theta}}{\rho_{0} J_{\Theta}^{2}}\right) \dot{\Theta}+2 \rho \sum_{i=1}^{N}\left(\overline{\mathbf{B}}_{\mathbf{e}}^{i} \frac{\partial \varphi}{\partial \overline{\mathbf{B}}_{\mathbf{e}}^{i}}\right): \overline{\mathbf{D}}_{\mathbf{v}}^{\mathbf{o} i} \\
-\rho\left(\frac{\partial \varphi}{\partial q}-\frac{\left(1-J_{m}\right)}{\rho_{0}}\right) \dot{q}-\rho \sum_{i=1} \frac{\partial \varphi}{\partial \omega_{i}} \dot{\omega}_{i}-\rho \frac{\partial \varphi}{\partial \xi} \dot{\xi} \\
-\frac{\operatorname{grad}_{\boldsymbol{x}} \Theta}{\Theta} \mathbf{q}_{\Theta} \geq 0 \quad \forall \mathbf{D}, \dot{\Theta}, \overline{\mathbf{D}}_{\mathbf{v}}^{\mathbf{o} i}, \dot{q}, \mathbf{q}_{\Theta}, \dot{\omega}_{i}, \dot{\xi}
\end{gathered}
$$

where $\phi$ is the total dissipation, $\boldsymbol{\sigma}$ is the Cauchy stress, $\mathbf{D}$ is the Eulerian strain rate: $\mathbf{D}=1 / 2\left(\mathbf{L}+\mathbf{L}^{\mathrm{T}}\right)$ with $\mathbf{L}=\dot{\mathbf{F F}}^{-1}, \rho, \rho_{0}$ are the current and initial densities, $s$ is the specific entropy, $\mathbf{q}_{\Theta}$ is the Eulerian heat flux, $\overline{\mathbf{D}}_{\mathbf{v}}^{\mathbf{o} i}$ is the $i^{t h}$ objective Eulerian strain rate, defined from:

$$
\overline{\mathbf{D}}_{\mathbf{v}}^{\mathbf{o}^{i}}=\overline{\mathbf{R}}_{\mathbf{e}}^{i} \overline{\mathbf{D}}_{\mathbf{v}}^{i} \overline{\mathbf{R}}_{\mathbf{e}}^{i^{\mathrm{T}}}
$$

where $\overline{\mathbf{R}}_{\mathbf{e}}^{i}$ comes from the polar decomposition $\overline{\mathbf{F}}_{\mathbf{e}}^{i}=$ $\overline{\mathbf{V}}_{\mathbf{e}}^{i} \overline{\mathbf{R}}_{\mathbf{e}}^{i}$. Assuming that the dissipation is only due to thermal diffusion and to the evolution of internal variables related to viscosity, Payne effect and chemical reactions, we can obtain the following constitutive equations from eq. (5):

$$
\boldsymbol{\sigma}=\overbrace{2 \rho\left(\overline{\mathbf{B}} \frac{\partial \varphi_{i s o}}{\partial \overline{\mathbf{B}}}\right)^{\mathrm{D}}+\frac{q}{J_{\Theta}} \mathbf{1}}^{\boldsymbol{\sigma}_{e q}}+\sum_{i=1}^{N} \overbrace{2 \rho\left(\overline{\mathbf{B}}_{\mathbf{e}}^{i} \frac{\partial \varphi_{i s o}}{\partial \overline{\mathbf{B}}_{\mathbf{e}}^{i}}\right)^{\mathrm{D}}}^{\boldsymbol{\sigma}_{v}^{i}}
$$

$s=-\frac{\partial \varphi}{\partial \Theta}+\frac{q}{\rho_{0}} \frac{J \alpha_{\Theta}}{J_{\Theta}^{2}}$

$\frac{\partial \varphi}{\partial q}=\frac{\left(1-J_{m}\right)}{\rho_{0}}$

where $\sigma_{e q}$ is the equilibrium stress (relaxed configuration), $\boldsymbol{\sigma}_{v}^{i}$ is the $i^{t h}$ viscous (non-equilibrium) stress. Equation (9) is a constitutive equation for $q$ and we can see from eq. (7) that the hydrostatic pressure is 
defined from $p=q / J_{\Theta}$. The remaining terms in the Clausius-Duhem inequality can be shortened as:

$\phi=\sum_{i=1}^{N} \phi_{\text {vis }}^{i}+\sum_{i=1}^{N} \phi_{\text {payne }}^{i}+\phi_{\xi}+\phi_{\Theta} \geq 0$

We assume that these remaining terms are independantly positives and we can easly satisfy the positivness of the heat conduction term $\left(\phi_{\Theta}\right)$ by choosing a standard isotropic Fourier law: $\mathbf{q}_{\Theta}=-k_{\Theta} \operatorname{grad}_{x} \Theta$ where the thermal conductivity parameter, $k_{\Theta}$, is assumed as independant of ageing.

The conservation of energy can be written as follows in the current configuration:

$\rho \Theta \dot{s}=\sum_{i=1}^{N} \phi_{\text {vis }}^{i}+\sum_{i=1}^{N} \phi_{\text {payne }}^{i}+\phi_{\xi}+\rho r-\operatorname{div}_{\mathbf{x}} \mathbf{q}_{\Theta}$

where $\operatorname{div}_{\mathbf{x}}$ is the Eulerian divergence, $r$ is an external heat supply term defined by unit of mass. Computing the material time derivative of entropy from its constitutive equation, eq. (8), we can obtain the following local form of the heat balance:

$$
\begin{aligned}
\rho C_{p} \dot{\Theta} & =\sum_{i=1}^{N} \phi_{v i s}^{i}+\sum_{i=1}^{N} \phi_{\text {payne }}^{i}+\phi_{\xi}+\rho r+l_{m}+l_{q} \\
& +l_{\xi}-\operatorname{div}_{\mathbf{x}} \mathbf{q}_{\Theta}
\end{aligned}
$$

where $C_{p}$ is the isobaric heat capacity:

$C_{p}=-\Theta\left(\frac{\partial^{2} \varphi}{\partial \Theta^{2}}+\frac{2 J q \alpha_{\Theta}^{2}}{J_{\Theta}^{3} \rho_{0}}\right)$

the coupling terms $l_{m}, l_{q}$ and $l_{\xi}$ are defined from:

$$
\begin{aligned}
& l_{m}=\Theta \frac{\partial \boldsymbol{\sigma}}{\partial \Theta}: \mathbf{D}-\Theta \sum_{i=1}^{N} \frac{\partial \boldsymbol{\sigma}_{v}^{i}}{\partial \Theta}: \overline{\mathbf{D}}_{\mathbf{v}}^{\mathbf{o}^{i}}+\Theta \sum_{i=1}^{N} \frac{\partial A_{\omega}}{\partial \Theta} \dot{\omega}_{i} \\
& l_{q}=-\Theta \frac{\alpha_{\Theta}}{J_{\Theta}^{2}} \dot{q} \\
& l_{\xi}=\frac{\partial A_{\xi}}{\partial \Theta} \dot{\xi}
\end{aligned}
$$

where $A_{\omega}, A_{\xi}$ are the thermodynamical forces associated with Payne effect or thermal ageing $\left(A_{\omega}=\right.$ $\left.-\rho \partial \varphi_{i s o} / \partial \omega^{i}, A_{\xi}=-\rho \partial \varphi / \partial \xi\right)$

\subsection{A thermo-chemo-viscoelastic model}

Considering the case of isotropic behaviors we propose to adopt the following potentials:

$$
\begin{aligned}
& \rho_{0} \varphi_{\text {iso }}=c_{10}(\xi, \Theta)\left(I_{1}(\overline{\mathbf{B}})-3\right)+c_{01}(\xi, \Theta)\left(I_{2}(\overline{\mathbf{B}})-3\right) \\
& +\sum_{i=1}^{N} \omega_{i} \frac{\mu_{i}(\xi, \Theta)}{2}\left(I_{1}\left(\overline{\mathbf{B}}_{\mathbf{e}}^{i}\right)-3\right) \\
& \rho_{0} \varphi_{\text {therm }}=C_{0}\left(\Theta-\Theta_{0}-\Theta \log \left(\frac{\Theta}{\Theta_{0}}\right)\right) \\
& -C_{1} \frac{\left(\Theta-\Theta_{0}\right)^{2}}{2 \Theta_{0}} \\
& \rho_{0} \varphi_{\text {chem }}=C_{2}\left(\tanh \left(m \frac{\Theta-\Theta_{\text {ind }}}{\Theta_{\text {ind }}}\right) \frac{(1-\xi)^{n+1}}{n+1}\right. \\
& \left.-\tanh \left(m \frac{\Theta_{0}-\Theta_{\text {ind }}}{\Theta_{\text {ind }}}\right)\right) \\
& \rho_{0} \varphi_{\text {vol }}=-k\left(\exp \left(\frac{q}{k}\right)\right)+q+k
\end{aligned}
$$

where $c_{10}, c_{20}$ are the Mooney-Rivlin coefficients that are assumed to be dependent on $\xi$ and $\Theta, \mu_{i}$ is the $i^{t h}$ viscous shear modulus that depends on $\xi$ and $\Theta$. The bulk modulus, $k$, is assumed to be independent of these variables. $I_{1}$ and $I_{2}$ are respectively the first and the second invariant of a strain tensor. $C_{0}$ and $C_{1}$ define the isobaric heat capacity (which is assumed to be only temperature dependent in this paper), $C_{2}$ is a heat-like coefficient related to the chemical process, $\Theta_{\text {ind }}$ is an induction temperature for the chemical process $^{2}, n$ and $m$ are chemical (ageing) parameters.

Using the previous definition, from eqs. (7) and (9), we can obtain the equilibrium stress and compressibility law:

$$
\begin{aligned}
\boldsymbol{\sigma}_{e q}= & 2 J^{-1} c_{10}(\xi, \Theta) \overline{\mathbf{B}}^{\mathrm{D}}+2 J^{-1} c_{01}(\xi, \Theta)\left(I_{1}(\overline{\mathbf{B}}) \overline{\mathbf{B}}\right. \\
& \left.-\overline{\mathbf{B}}^{2}\right)^{\mathrm{D}}+\frac{q}{J_{\Theta}} \mathbf{1} \\
J_{m}= & \exp \left(\frac{q}{k}\right)
\end{aligned}
$$

From eq. (7), we can calculate the $i^{t h}$ viscous stress, which is defined by:

$$
\boldsymbol{\sigma}_{v}^{i}=J^{-1} \mu_{i}(\xi, \Theta) \omega_{i} \overline{\mathbf{B}}_{\mathbf{e}}^{i} \mathrm{D} \quad i=1 . . N
$$

\footnotetext{
${ }^{2}$ No chemical reactions are assumed to occur if $\Theta<\Theta_{\text {ind }}$
} 
We consider a Maxwell-like flow rule for viscosity as follows:

$\overline{\mathbf{D}}_{\mathbf{v}}^{\mathbf{o}^{i}}=\frac{1}{\eta_{i}\left(\xi, \Theta, \omega_{i}\right)} \boldsymbol{\sigma}_{v}^{i} \quad i=1 . . N$

where $\eta_{i}\left(\xi, \Theta, \omega_{i}\right)$ is the $i^{\text {th }}$ viscosity parameter. The Maxwell flow rule can also be also reformulated as follows:

$\dot{\overline{\mathbf{B}}}_{\mathbf{e}}{ }^{i}=\mathbf{L} \overline{\mathbf{B}}_{\mathbf{e}}^{i}+\overline{\mathbf{B}}_{\mathbf{e}}^{i} \mathbf{L}^{\mathrm{T}}-\frac{2}{3}(\mathbf{1}: \mathbf{L}) \overline{\mathbf{B}}_{\mathbf{e}}^{i}-\frac{1}{J \tau_{i}} \overline{\mathbf{B}}_{\mathbf{e}}^{i} \overline{\mathbf{B}}_{\mathbf{e}}^{i \quad \mathrm{D}}$

$\overline{\mathbf{B}}_{\mathbf{e}}^{i}(t=0)=\mathbf{1}, \quad i=1 . . N$

where $\tau_{i}=\eta_{i} / 2 \omega_{i} \mu_{i}$ is the $i^{t h}$ characteristic time of viscosity.

For the Payne effect, we adopt the same model as already proposed in Delattre, Lejeunes, Lacroix, \& Méo 2016:

$$
\begin{gathered}
\dot{\omega}_{i}=-\frac{1}{h_{i}}\left\langle\omega_{i}-\left(\frac{3}{I_{1}(\overline{\mathbf{B}})}\right)^{r_{i}}\right\rangle \\
\omega_{i}(t=0)=1 \quad i=1 . . N
\end{gathered}
$$

where $h_{i}$ is the $i^{\text {th }}$ intrinsic time for Payne effect and $r_{i}$ is a dimensionless material parameter. These parameters and the characteristic times of viscosity $\left(\tau_{i}\right)$ are assumed to be independant of ageing and temperature. However as the viscous shear modulus depend on ageing and temperature, viscosity in this model is dependant on these parameters.

For the chemical evolution due to thermal ageing, we can first compute the thermodynamic force associated with the chemical aging, $A_{\xi}$ is given by:

$$
\begin{aligned}
A_{\xi}= & J^{-1} C_{2} \tanh \left(m \frac{\Theta-\Theta_{i n d}}{\Theta_{i n d}}\right)(1-\xi)^{n} \\
& -J^{-1} \frac{\partial c_{10}}{\partial \xi}\left(I_{1}(\overline{\mathbf{B}})-3\right)-J^{-1} \frac{\partial c_{01}}{\partial \xi}\left(I_{2}(\overline{\mathbf{B}})-3\right) \\
& -J^{-1} \sum_{i=1}^{N} \omega_{i} \frac{1}{2} \frac{\partial \mu_{i}}{\partial \xi}\left(I_{1}\left(\overline{\mathbf{B}}_{\mathbf{e}}^{i}\right)-3\right)
\end{aligned}
$$

As in (Lejeunes, Eyheramendy, Boukamel, Delattre, Méo, \& Ahose 2018) we propose the following chemical evolution equation (that fullfills thermodynamical requirements):

$\dot{\xi}=A \exp ^{-\frac{E_{a}}{R \Theta}}\left\langle A_{\xi}\right\rangle$ where $\langle$.$\rangle are the so called Macaulay brackets,$ $R=8.314 \mathrm{~J} / \mathrm{mol} / \mathrm{K}$ is the ideal gas constant, $E_{a}$ is an activation energy and $A$ is a kinetic parameter (unit of $\mathrm{Pa}^{-1} \mathrm{~s}^{-1}$ ). Using eq. (21) in eq. (22) it can be clearly seen that the proposed model takes into account of a chemo-mechanical coupling effect. Only the isochoric part can play a role on chemical evolution but the hybrid energy approach allows to easly include a volumetric (pressure) dependance if needed (this have been proposed in (Lejeunes, Eyheramendy, Boukamel, Delattre, Méo, \& Ahose 2018)). If we consider stress-free ageing, the chemical evolution reduces to:

$$
\dot{\xi}=J_{\Theta}^{-1} A C_{2} \exp ^{-\frac{E_{a}}{R \Theta}}\left\langle\tanh \left(m \frac{\Theta-\Theta_{i n d}}{\Theta_{\text {ind }}}\right)\right\rangle(1-\xi)^{n}
$$

Equation (23) defines a non Arrhenius behavior when $\Theta$ is lower than or close to the induction temperature and tends to Arrhenius behavior when $\Theta$ is higher than the induction temperature.

\section{FINITE ELEMENT IMPLEMENTATION}

For the finite element implementation, we consider the simultaneous resolution of quasi-static mechanical equilibrium, energy conservation, chemical evolution and compressibility law. We can therefore establish a four fields formulation that can be writen as follows in a Lagrangian (first Piola-Kirchhoff) configuration:

$\operatorname{Find}(\mathbf{u}, q, \Theta, \xi), \quad$ such that $\forall(\delta \mathbf{u}, \delta q, \delta \Theta, \delta \xi)$

$$
\begin{gathered}
\int_{\Omega_{0}}\left(\boldsymbol{\Pi}_{e q}^{D E V}+\sum_{i=1}^{N} \boldsymbol{\Pi}_{v}^{i}+q \frac{J}{J_{\Theta}} \mathbf{F}^{-\mathrm{T}}\right): \mathbf{F}(\delta \mathbf{u}) d \Omega_{0} \\
\quad-\int_{\Omega_{0}} \rho_{0} \mathbf{f} \delta \mathbf{u} d \Omega_{0}-\int_{\partial \Omega_{f}} \mathbf{T} \delta \mathbf{u} d S=0 \\
\int_{\Omega_{0}} \rho_{0} C_{p} \dot{\Theta} \delta \Theta d \Omega_{0}-\int_{\Omega_{0}}\left(J \sum_{i=1}^{N} \phi_{v i s}^{i}+J \sum_{i=1}^{N} \phi_{\text {payne }}^{i}\right. \\
\left.+J \phi_{\xi}+\rho_{0} r+J l_{m}+J l_{q}+J l_{\xi}\right) \delta \Theta d \Omega_{0} \\
-\int_{\Omega_{0}} \mathbf{Q}_{\Theta} \mathrm{GRAD}_{\mathbf{X}} \delta \Theta d \Omega_{0}+\int_{\partial \Omega_{Q}} \mathbf{Q}_{0} \delta \Theta d S=0
\end{gathered}
$$

$\int_{\Omega_{0}}\left(\frac{J}{J_{\Theta}}-\exp \left(\frac{q}{k}\right)\right) \delta q d \Omega_{0}=0$

$\int_{\Omega_{0}}\left(\dot{\xi}-k_{\xi}(\Theta)\left\langle A_{\xi}\right\rangle\right) \delta \xi d \Omega_{0}=0$ 
where $\Pi_{e q}^{D E V}=J \boldsymbol{\sigma}_{e q}^{\mathrm{D}} \mathbf{F}^{-T}, \boldsymbol{\Pi}_{v}^{i}=J \boldsymbol{\sigma}_{v}^{i} \mathbf{F}^{-T}$ and $\mathbf{Q}_{\Theta}=$ $J \mathbf{q}_{\Theta} \mathbf{F}^{-T}$. To resolve the previous non-linear system, we firt consider a backward Euler scheme to integrate the system with respect to time. The time step is defined as $\Delta t=\left(t_{n}, t_{n+1}\right)$ and we use the following approximations: $\dot{\Theta}=\left(\Theta^{n+1}-\Theta^{n}\right) / \Delta t$ and $\dot{\xi}=$ $\left(\xi^{n+1}-\xi^{n}\right) / \Delta t$. We can then linearize the coupled system into a Newton-Raphson scheme. We obtain a non-symetric tangent but we can take into account strong coupling effects in a simple formulation and a simple numerical implementation. The constitutive relations for the stress and the evolution equations for viscosity (19) or for Payne (20) are locally integrated at each Gauss point with substepping backward Euler schemes (e.g. Lejeunes, Boukamel, \& Méo 2011). We integrated all these developments within the simulation platform FEMJava that is being developed in our laboratory (see for instance Eyheramendy, Lejeunes, Saad, \& Zhang 2014).

\section{MATERIAL PARAMETER IDENTIFICATION}

To identiffy the material parameters, we proceeded with the following steps:

1. Identification of the chemical paramters $m, n, E_{a}, \Theta_{i n d}, Q_{\infty}$ and the product $A C_{2}$ from the results of swelling tests on samples aged in stress-free and anaerobic conditions.

2. Identification of hyperelastic parameters $c_{10}(\xi, \Theta), c_{01}(\xi, \Theta)$ from tensile tests performed on virgin samples at different temperatures and aged samples at different aging time (but characterized at room temperature).

3. Identification of the viscoelastic and softening parameters $\mu_{i}(\xi, \Theta), \tau_{i}, h_{i}, r_{i}, N$ with a strategy similar to that presented in Delattre, Lejeunes, Méo, Lacroix, \& Richard 2014. As for the hyperelastic test, this identification is based on tensile tests performed on virgin samples at different temperatures and aged samples at different aging time (but again characterized at room temperature).

4. Identification of other parameters: compressibility $k$ from oedometric tests, heat capacity parameter $C_{1}, C_{0}$ from DSC tests, thermal expansion $\alpha_{\Theta}$ from dilatometry experiments. The thermal conductivity parameter, $k_{\Theta}$, is taken from the literature.

5. Identification of $A$ and $C_{2}$ from inhomogeneous creep aging tests by inverse finite element simulations of the creep aging test.

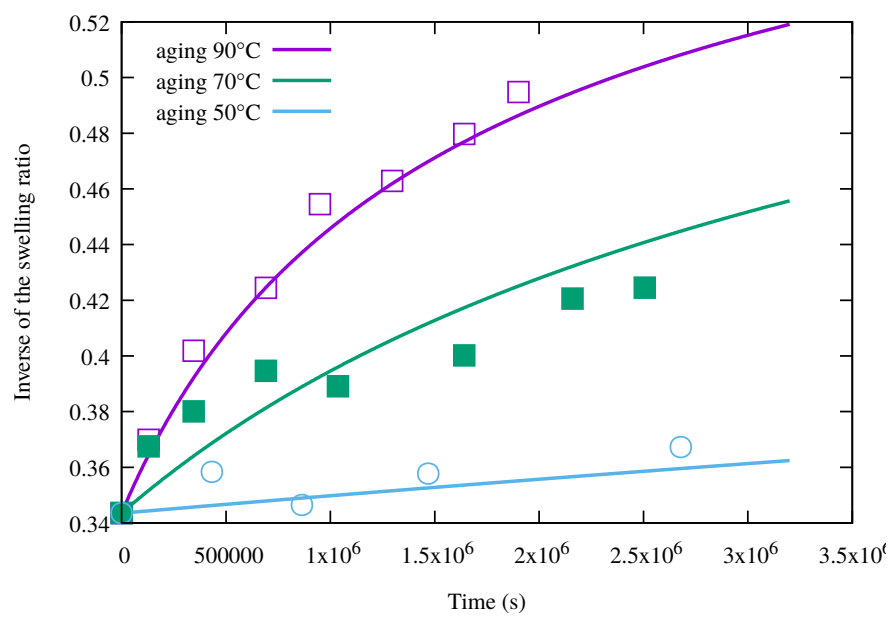

Figure 1: Model/experiments comparison for the chemical part of the model after step 1 of the identification process (experiments are represented by square or circle dots, model by continuous lines)

Whether for swelling tests or mechanical tests, we repeated the experiments at least twice on different samples and calculate an average response (for swelling or tensile experiment). We assumed a linear dependancy for the mechanical moduli such as (same hypothesis for $\left.c_{10}(\xi, \Theta), c_{01}(\xi, \Theta)\right)$ :

$\mu_{i}(\xi, \Theta)=\mu_{i}^{a} \xi+\mu_{i}^{b} \frac{\Theta-\Theta_{0}}{\Theta_{0}}+\mu_{i}^{0}$

The results of the identification steps are given here after. Figure 1 shows the results obtained after step 1. For this identification we have arbitraly fixed the induction temperature such that: $\Theta_{\text {ind }}=320 \mathrm{~K}$.

The figures 2 and 3 illustrate the results obtained after identifying the mechanical part of the model (step 2 and 3). After step 4 of the identification, finite element simulations of creep aging test were performed: we considered a FE model of a tensile sample under permenant load and imposed volumetric heating and adiabatic boundary conditions such as to obtain a homogeneous and stable temperature in the sample. These boundary and loading conditions allowed the thermal regulation in the oven to be reproduce even if experimental conditions are not strictly the same. Figures 5 show a tipical result obtained from this simulation. It can be seen that if the temperature field is homogeneous (cf. figure 5(a)), this is not the case of the chemical aging field (cf. figure 5(c)) due to the nonstress free conditons. By analysing the FE results and calculating the error between the experimental results, we performed an inverse identification of the chemomechanical coupling parameter (step 5 of the identification).

Figure 4 shows some iterations of this inverse identification. It can be seen from this results that, as expected, for a fixed value of the product $A C_{2}$ previously identified from step 1 , if $C_{2}$ is high (or $A$ is low) the influence of the stress field is negligeable and when $C_{2}$ is low (or $A$ is high) the stress field penalizes the chemical evolution. The table 1 summarizes 


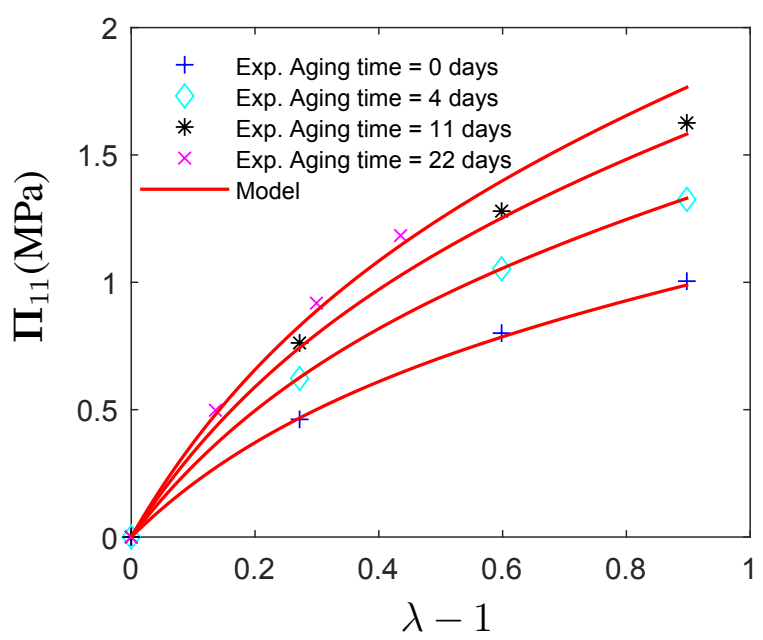

(a) Aging time dependency (mechanical characterisation at room temperature, stress free aging at $90^{\circ} \mathrm{C}$ )

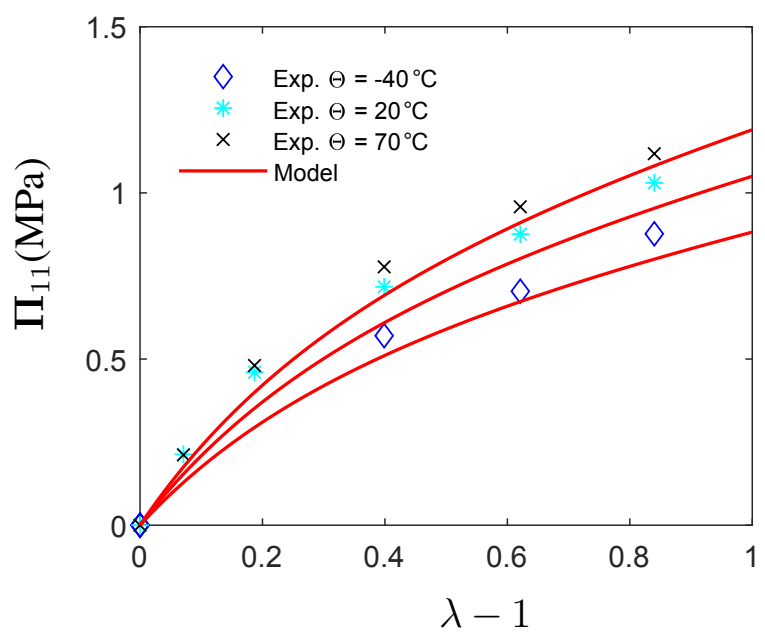

(b) Temperature dependency (virgin state)

Figure 2: Model/experiments comparison for the hyperelastic part of the model (equilibrium responses) at different temperature and different aging time after step 2 of the identification

the material parameters obtained from the identification process.

\section{CONCLUSION}

Modeling and simulation of the thermal ageing of elastomers and its consequences on the behavior of the material is very challenging due to the strong coupling phenomena that occur during aging. In addition, current experimental techniques do not allow precise access to the physics of network evolution. Therefore, only phenomenological approaches such as the one proposed in this work can be used to describe ageing. In many industrial applications, ageing is not homogeneous due to the presence of simultaneaous mechnical loads. Thus, the chemo-mechanical coupling (aging under non stress-free conditions) must be taken into account in the modeling. In this work, we have proposed a modeling approach that takes

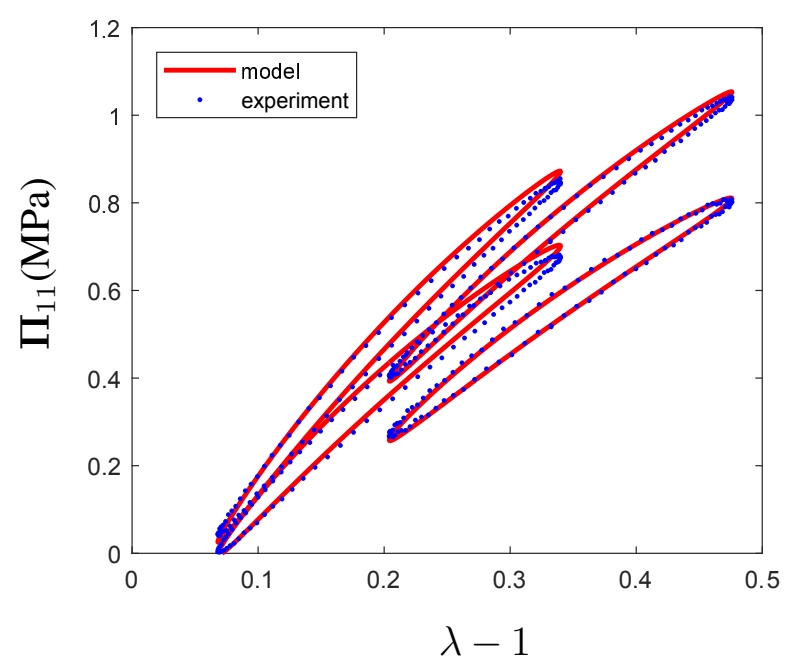

(a) Dynamic amplitude $=0.136$, Aging time $=$ 8 days, frequency $f=10 \mathrm{~Hz}$, ageing temperature $=90^{\circ} \mathrm{C}$

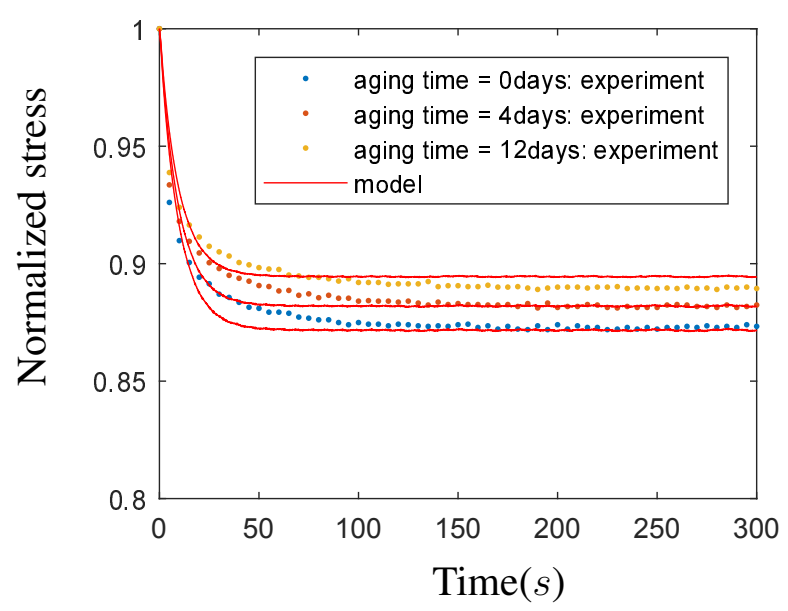

(b) Relaxation curves for samples aged at $70^{\circ} \mathrm{C}$

Figure 3: Model/experiments comparison of the model at different temperature and different aging time after step 3 of the identification

into account: nonlinear viscosity (dynamical softening), thermo-mechanical coupling (entropic behavior for the fully relaxed behavior and thermal softening for the viscosity and thermal expansion) and thermo-chemo-mechanical couplings (ageing under non-stress free condtions). We have also proposed an identification strategy to determine material parameters. The finite element implementation of this model within a fully thermo-chemo-mechanical framwork have allowed us to use inverse FE simulation for indentification. This FE implementation will be used in a future work to confront the model with other experimental results that will be otained in more complex situations (e.g. with cyclic mechanical and thermal loads).

\section{REFERENCES}

Ahose, K. D. (2018). Characterization and modeling of thermal ageing of elastomers by a multiphysics approach. $\mathrm{Ph} . \mathrm{D}$. the- 


\begin{tabular}{|c|c|c|c|}
\hline & \multirow{2}{*}{\multicolumn{2}{|c|}{$E_{a}(J / m o l)$}} & \\
\hline & & & $\begin{array}{c}51000 \\
32\end{array}$ \\
\hline & \multicolumn{2}{|c|}{$m$} & 232 \\
\hline & \multicolumn{2}{|c|}{$A\left(M P a^{-1} s^{-1}\right)$} & $\begin{array}{l}2.140 \\
8.17\end{array}$ \\
\hline & \multicolumn{2}{|c|}{$C_{2}(M P a)$} & 1.43 \\
\hline & \multicolumn{2}{|c|}{$Q_{\infty}$} & 0.658 \\
\hline & \multicolumn{2}{|c|}{$\Theta_{\text {ind }}(K)$} & 320 \\
\hline & \multicolumn{2}{|c|}{$c_{10}(M P a)$} & $0.37 \xi+0.146 \frac{\Theta-\Theta_{0}}{\Theta_{\rho}}+0.2$ \\
\hline & \multicolumn{2}{|c|}{$c_{01}(\xi, \Theta)(M P a)$} & $0.32 \xi+0.175 \frac{\Theta-\Theta_{0}}{\Theta}+0.2$ \\
\hline & \multicolumn{2}{|c|}{$k(M P a)$} & $1400^{0}$ \\
\hline & \multicolumn{2}{|c|}{$\alpha_{\Theta}\left(K^{-1}\right)$} & $6 . e-4$ \\
\hline & \multicolumn{2}{|c|}{$C_{0}\left(N m m^{-2} K^{-1}\right)$} & 4.6 \\
\hline & \multicolumn{2}{|c|}{$C_{1}\left(N m m^{-2} K^{-1}\right)$} & 0.39 \\
\hline & \multicolumn{2}{|c|}{$k_{\Theta}\left(N s^{-1} K^{-1}\right)$} & 0.22 \\
\hline$\tau_{i}(s)$ & 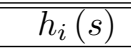 & $r_{i}$ & $\mu_{i}(\xi, \Theta)(M P a)$ \\
\hline 0.01 & 1 & 0.05 & $-0.0365 \xi-0.0586 \frac{\Theta-\Theta_{0}}{\Theta_{0}}+0.1167$ \\
\hline 0.01 & 1 & 5 & $-0.0448 \xi^{0}$ \\
\hline 0.1 & 1 & 0.05 & $-0.0016 \xi+0.0836$ \\
\hline 0.1 & 1 & 5 & $-0.0499 \xi$ \\
\hline 1 & 1 & 0.05 & $-0.0039 \xi-0.3811 \frac{\Theta-\Theta_{0}}{\Theta_{0}}+0.0110$ \\
\hline 1 & 1 & 5 & $-0.0119 \xi^{0}$ \\
\hline 10 & 1 & 0.05 & $-0.0586 \frac{\Theta-\Theta_{0}}{\Theta_{0}}+0.1415$ \\
\hline 10 & 1 & 5 & $-0.1759 \frac{\Theta-\Theta_{0}}{\Theta_{0}}$ \\
\hline
\end{tabular}

Table 1: Material parameters

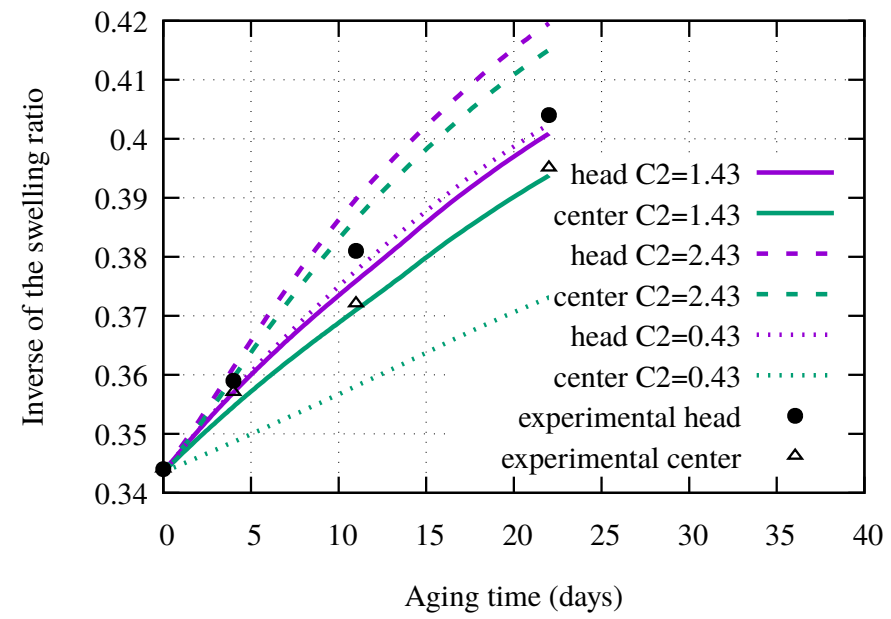

Figure 4: Comparison of numerical simulation and experimental results for the thermal creep aging test. The swelling ratio is determined experimentally and numerically in two zones of the tensile samples: center, for the central zone where stress is maximum (in red in figure 5(b)), head, that correspond to a zone where cross section is high and stress is minimum (in blue in figure 5(b)).

sis, Ecole Centrale Marseille.

Ahose, K. D., S. Lejeunes, D. Eyheramendy, \& F. Sosson (2017). On the thermal aging of a filled butadiene rubber. In A. Lion and M. Johlitz (Eds.), Proceedings of the European Conference on Constitutive Models for Rubbers X, pp. 59-64. Taylor \& Francis.

Delattre, A., S. Lejeunes, F. Lacroix, \& S. Méo (2016). On the dynamical behavior of filled rubbers at different temperatures : Experimental characterization and constitutive modeling. International Journal of Solids and Structures 90, 178-193.

Delattre, A., S. Lejeunes, S. Méo, F. Lacroix, \& C. Richard (2014). On the multiaxial amplitude and frequency dependent behavior of rubber: experiments and constitutive modeling. Rubber Chemistry and Technology 87(3), 557-578.

Eyheramendy, D., S. Lejeunes, R. Saad, \& L. Zhang (2014). Advances in symbolic and numerical approaches in computational mechanics. In B. H. V. Topping and P. Iványi (Eds.), Computational Methods for Engineering Technology, Chap-

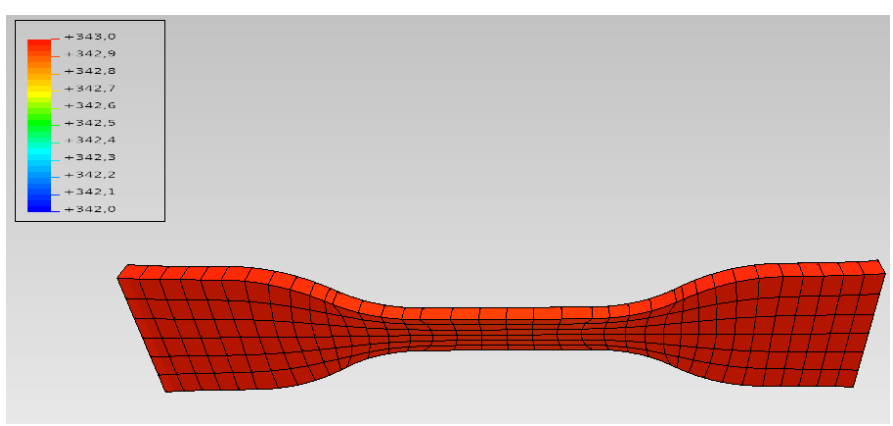

(a) Temperature field in Kelvin at the end of the heating and mechanical loading phase

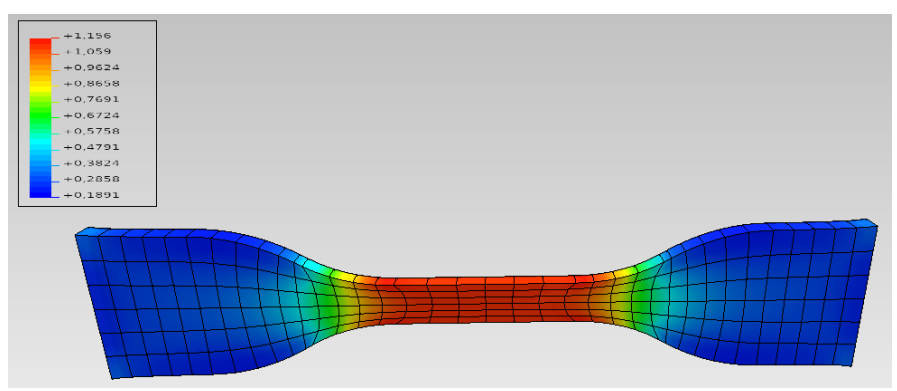

(b) Axial stress field (Cauchy stress in MPa) at the end of the heating and mechanical loading phase

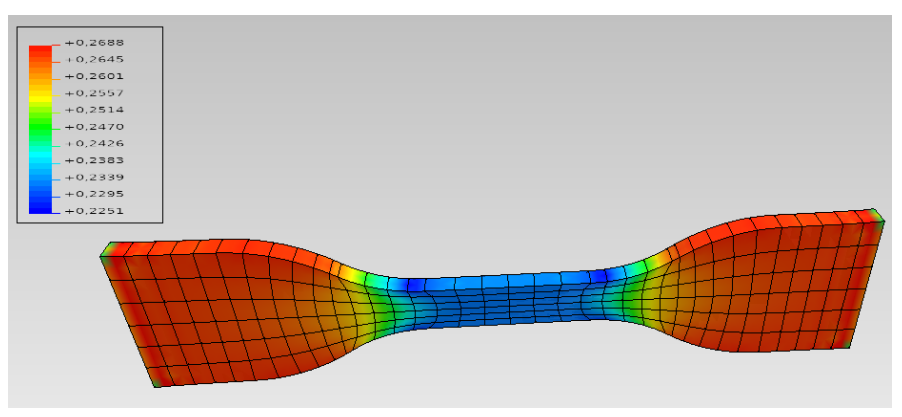

(c) Chemical conversion field after 22 aging days

Figure 5: FE simulation results of the numerical creep aging test (for $C_{2}=1.43 \mathrm{MPa}$ and $A=8.17 \mathrm{MPa}^{-1} \mathrm{~s}^{-1}$ )

ter 3, pp. 61-88. Saxe-Coburg Publications.

Kari, L. (2017, Sep). Dynamic stiffness of chemically and physically ageing rubber vibration isolators in the audible frequency range. Continuum Mechanics and Thermodynamics 29(5), 1027-1046.

Lejeunes, S., A. Boukamel, \& S. Méo (2011). Finite element implementation of nearly-incompressible rheological models based on multiplicative decompositions. Computers \& Structures 89(3), $411-421$.

Lejeunes, S. \& D. Eyheramendy (2018, May). Hybrid free energy approach for nearly incompressible behaviors at finite strain. Continuum Mechanics and Thermodynamics.

Lejeunes, S., D. Eyheramendy, A. Boukamel, A. Delattre, S. Méo, \& K. D. Ahose (2018, Feb). A constitutive multiphysics modeling for nearly incompressible dissipative materials: application to thermo-chemo-mechanical aging of rubbers. Mechanics of Time-Dependent Materials 22(1), 51-66.

Musil, B., M. Johlitz, \& A. Lion (2018, Oct). On the ageing behaviour of nbr: chemomechanical experiments, modelling and simulation of tension set. Continuum Mechanics and Thermodynamics. 